G OPEN ACCESS

\section{Paracentesis Simulation: A Comprehensive Approach to Procedural Education}

Dana Sall, MD, MEd, Gregory W. Wigger, MD*, Benjamin Kinnear, MD, Matthew Kelleher, MD, MEd, Eric Warm, MD, Jennifer K. O'Toole, MD, MEd

*Corresponding author: gregory.wigger@uc.edu

\section{Abstract}

Introduction: Structured procedural education and assessment of competency are growing needs for residency and fellowship programs. Simulation is a useful way to learn, experience, and practice procedural skills with competence. Paracentesis is a common procedure encountered in internal medicine. This educational resource for paracentesis education includes didactics, cases, and assessments to address cognitive skills, a simulation experience to address psychomotor procedural skills, and an entrustment-based assessment tool. Methods: Prior to the simulation, learners completed preprocedural didactics and self-assessments. Utilizing a paracentesis trainer, ultrasound, and paracentesis kit, the case of a 46-year-old male with ascites in need of a paracentesis was presented. During the simulation, learners initially performed a paracentesis step by step, with assistance and feedback from the case instructor. This was immediately followed by paracentesis without assistance, where the instructor evaluated the learners with an assessment tool encompassing a procedural checklist, global skill assessment scale, and entrustment scale. Afterwards, learners completed case-based reviews and returned to the simulation lab several months later to repeat an unassisted paracentesis. Results: The curriculum was used with internal medicine and medicine-pediatric residents of all training levels. To date, over 120 residents have completed the curriculum. Residents reported an increase in self-confidence and competence using ultrasound to identify ascites and performing a paracentesis. Learners provided positive feedback. Discussion: This curriculum offers the opportunity for both cognitive and psychomotor paracentesis education in a low-risk simulation environment. The comprehensive strategy with didactics, cases, and multiple simulations is designed to promote knowledge and skill retention.

\section{Keywords}

Editor's Choice, Simulation, Paracentesis, Ascites, Procedural Education

\section{Educational Objectives}

By the end of this session, learners will be able to:

1. Demonstrate informed consent for a simulated paracentesis procedure.

2. Perform a simulated diagnostic and therapeutic paracentesis procedure.

3. Demonstrate the use of ultrasound as it pertains to performing a paracentesis.

4. Incorporate feedback to improve paracentesis performance.

5. Interpret laboratory values to diagnose the etiology of ascites.

6. Diagnose and treat spontaneous bacterial peritonitis.

\section{Introduction}

There is a growing interest surrounding internal medicine residency training and procedural education. Today's internists are performing fewer bedside procedures than prior generations. ${ }^{1}$ A host of concerns, including those for patient safety and lack of consistent training, evaluation, and feedback, has placed the common "see one, do one, teach one" methodology under significant scrutiny. ${ }^{2}$ The American Board of Internal Medicine (ABIM) requires all residents to demonstrate core manual skills in procedures such as advanced cardiac life support, drawing venous and arterial blood, placing a peripheral IV, and performing a Pap smear and endocervical culture. The ABIM also requires competence with regard to knowledge and
Citation: Sall D, Wigger GW, Kinnear B, Kelleher M, Warm E, O'Toole JK. Paracentesis simulation: a comprehensive approach to procedural education. MedEdPORTAL. 2018;14:10747.

https://doi.org/10.15766/mep_23748265.10747

Copyright: $\odot 2018$ Sall et al. This is an open-access publication distributed under the terms of the Creative Commons Attribution-NonCommercialShare Alike license.

\section{Appendices}

A. Paracentesis Presentation .pptx

B. Instructional Ultrasound Presentation.pptx

C. Paracentesis Instructional Video.mp4

D. Ultrasound Assessment Quiz.docx

E. Paracentesis Assessment Quiz.docx

F. Paracentesis Checklist and Evaluation.docx

G. Simulation Case.docx

$\mathrm{H}$. Paracentesis Patient Prompt.docx

I. New Onset Ascites Case docx

J. Cirrhotic With Abdominal Pain Case.docx

All appendices are peer reviewed as integral parts of the Original Publication. 
understanding in several other invasive bedside procedures, including abdominal paracentesis. ${ }^{3}$ Since many trainees will also require skills in performing such procedures upon graduation, training curricula to address both cognitive and psychomotor learning of procedures like paracentesis are needed.

While didactics can help improve cognitive procedural skills, many programs lack adequate procedural frequency to improve psychomotor procedural skills. Simulation allows for both cognitive and integrative learning of a procedure in a low-risk environment and has been shown to improve residents' knowledge, skills, and confidence. ${ }^{4,5}$ In this way, simulation can help bridge the gap between infrequent procedural experience and improvement in skills. Furthermore, simulation can be used as a method of procedural skill maintenance, allowing learners to revisit and review procedure skills weeks to months after initial education-preventing the deterioration of procedural skills and clinical knowledge. An additional barrier to procedural education and training is the lack of a standard for assessing competency. ${ }^{6}$ Recent studies have attempted to address the lack of evaluation for competency by creating assessment tools for different bedside procedures. ${ }^{7-10}$ These tools differ in their use of assessment components such as checklists or global skills scales. It is still not clear which method of assessment is best linked to procedural competence while also permitting formative feedback. Some have suggested incorporating an entrustment scale into a standardized procedural assessment tool to help guide decisions of competency, ${ }^{9}$ but this type of tool did not exist for paracentesis.

We were motivated to create this curriculum after a patient presented with ascites and suffered a significant complication following a paracentesis. After review of the case, we noted a lack of standardized instruction, feedback, and assessment in regard to procedures, particularly with paracentesis. This gap in education was the nidus for our curriculum development. The target audience is internal medicine and medicine-pediatric residents of all years. While there were published resources, including in MedEdPORTAL, demonstrating paracentesis, ${ }^{11,12}$ none offered a comprehensive education. Thus, we created a comprehensive curricular package encompassing not only a simulation experience using deliberate practice to address psychomotor procedural skills but also didactics, cases and assessments to address cognitive skills, and an entrustment-based assessment tool to judge competence. This assessment tool, known as the Paracentesis Competency Assessment Tool (PCAT), combines a procedural skills checklist, a global skills scale, and an entrustment scale. The PCAT can be used for formative feedback and assessments of skill attainment on simulated and live patient procedures.

\section{Methods}

Development

Using the "learn, see, practice, prove, do, maintain" model, ${ }^{13}$ learners completed online comprehensive preprocedural education modules and assessments on paracentesis and ultrasound (learn), viewed a video of the procedure properly performed (see), practiced the procedure in a simulation laboratory with faculty guidance and deliberate practice (practice), and were assessed completing the simulated procedure (prove). Once a minimum standard of competence was demonstrated, residents were permitted to perform live paracentesis procedures with faculty supervision (do). Finally, residents returned to the simulation lab at varying intervals for continued practice and feedback and reviewed clinical pearls of the procedure in case reviews (maintain).

Internal medicine hospitalist faculty volunteered as case instructors. Prior to simulation, all instructors completed a training session that included a review of the procedural video while using the PCAT, a discussion of common learner mistakes and strategies for giving constructive feedback, and a debrief. Our faculty were familiar with the simulator and thus did not practice the procedure; however, if instructors are not familiar, then the training session should include an introduction to and guidance in use of the simulator.

Equipment/Environment

The simulated case was completed in a simulation lab using the following equipment: 
- Blue Phantom Paracentesis Ultrasound Training Model.

- SonoSite M-Turbo ultrasound machine.

- Tubing with connection to a large-volume suction collection container.

- Abdominal paracentesis kit: 10-cc sterile syringe, 35-cc sterile syringe, three needles (19-, 22-, and 25gauge), 5-cc ampule of lidocaine or saline, betadine swabs, 5.0 French/7-cm Yueh catheter, sterile drape, and sterile dressing.

- Sterile gloves, cap, mask.

- Gauze.

Personnel

The case instructor served multiple purposes throughout the simulation. Initially, he or she provided feedback and instruction to the learner during the initial run-through of the paracentesis. Later, the case instructor evaluated the learner's procedural performance. The case instructor played the patient role. The role included answering the learner's questions regarding the history as well as providing consent for the procedure.

Implementation Educational materials were first made available to the learners at the start of the academic year via our institution's online curriculum site, Blackboard. Each learner was notified 2 weeks prior to his or her planned simulation session and instructed to review these materials before attending. The paracentesis presentation (Appendix $A$ ) reviewed the basic steps of the procedure, including informed consent, the indications and contraindications for the procedure, and basic interpretation of laboratory values of ascitic fluid. The instructional ultrasound presentation (Appendix B) reviewed the basics of ultrasound technique and its usefulness specifically with the paracentesis procedure. A prerecorded video (Appendix C) reviewed a properly performed ultrasound-guided paracentesis from start to finish.

After completion of the educational materials, the learners completed assessments (ultrasound assessment quiz, Appendix D; paracentesis assessment quiz, Appendix E). Learners were then assigned time in the simulation lab. They performed the simulation individually with case instructors in order to provide ample time for questions, practice the procedure, and receive 1:1 feedback regarding deficiencies. Our learners were scheduled for 1-hour sessions. However, if time or space limits, simulation training could be completed in a small-group setting with up to four learners.

On arrival, each learner began by rewatching the procedural video at a computer in the simulation lab. While watching, the learner used the PCAT (Appendix F) to evaluate the video instructor's performance in order to familiarize him-/herself with the PCAT's criteria and begin to break the procedure down into concrete steps. During this time, the instructor set up the mannequin and the necessary equipment (Appendix G). If there is a need to do the training in a small-group setting, as discussed above, we recommend that learners watch the demonstration of the paracentesis as a group.

After the video, the learner read the prompt detailing the case of Mr. S (Appendix $H$ ) and began the paracentesis procedure on the mannequin, starting with informed consent. The instructor spoke for the patient as needed. The learner then performed the paracentesis step by step with the instructor, to ensure adequate understanding and skill at each step. The instructor stopped performance to assist or demonstrate at any time deemed necessary. Particular time was dedicated to ensuring the learner understood correct use of the ultrasound. This included proper orientation and placement of the ultrasound probe as well as interpretation and measurement of structures/organs seen with ultrasound related to paracentesis.

The learner practiced each step of the procedure as many times as he or she felt necessary to attain the skill. The instructor reviewed any missed or incorrectly performed steps of the procedure after the initial practice. At each point, the PCAT was used to provide assessment data and formative feedback so that 
the learner could incorporate and practice suggested changes in real time. If there is a need to do the training in a small-group setting, we still recommend that each learner perform the paracentesis with the instructor as discussed above. For time-saving purposes, the informed consent can be omitted after the first learner's demonstration. While one learner is performing the paracentesis, the remaining learners should observe and follow along with a PCAT as well. This will allow learners to learn from each other's mistakes and see solutions to commonly encountered problems. Due to the time needed to perform the procedure multiple times, we recommend increasing the simulation to a 90- to 120-minute session.

The instructor queried and discussed with the learner the types of studies the learner would send to evaluate for spontaneous bacterial peritonitis (PCAT step 25). Once the learner reported feeling comfortable with the procedure, the instructor immediately set up the mannequin for a repeated paracentesis. The learner then proceeded to a final attempt at an unassisted paracentesis from start to finish. The instructor evaluated the procedure with the PCAT. If there is a need to do the training in a small-group setting, each learner would need to perform the paracentesis individually. Since this is for an evaluation, we recommend that each learner be given the space individually, with colleagues outside of the room.

After completion of the procedure, the instructor reviewed any missed checklist items and deficiencies of the unassisted procedure. Learners were required to demonstrate a minimum level of competence (Minimum Passing Standard, described below) in order pass their simulation session. Learners who did not achieve this score on completion of their initial simulation session would have to return for a remediation session.

After initial training, learners returned to the simulation lab 3-6 months later with the same setup. Unlike the previously described simulation, learners did not watch a demonstration prior to this one. There was no extended teaching or practice of the procedure prior to the paracentesis. In this session, learners had 30 minutes to complete a paracentesis without the assistance of an instructor. The instructor first provided a brief prompt on the patient to the learner. The learner proceeded with the paracentesis procedure, beginning with informed consent. The instructor spoke for the patient as needed. During the procedure, the instructor evaluated the learner using the PCAT. After completion of the procedure, the instructor reviewed any missed checklist items, and time was given for the learner to repeat those steps.

In addition to the presimulation didactics and the simulation sessions, cases were created to review knowledge associated with the paracentesis procedure. Specific goals included the interpretation of diagnostic studies of ascitic fluid to determine its underlying etiology (Appendix I) and the diagnosis and treatment of spontaneous bacterial peritonitis (Appendix J). At our institution, these cases were taught at a weekly resident educational conference in a small-group setting of five to 10 residents per group several weeks after completion of the simulations. The delay was intended to provide spacing of knowledge learning in the presimulation didactics; however, these cases could also be reviewed immediately after the simulation or during a program's morning or noon conference following the simulation.

\section{Assessment}

Learners were assessed in multiple ways in this curriculum. After completion of the preprocedural educational materials, learners completed two quizzes covering information detailed in presentations to ensure adequate understanding of the material. A passing score was considered $>70 \%$. This was based on a group of medical education experts who gained group consensus for a minimum pass score of $70 \%$, a common passing score for standardized tests. During the procedure, instructors assessed learners using the PCAT (Appendix F). The first component of the PCAT is a skills checklist adapted, collated, and edited from other published paracentesis checklists. 5,9,14 The checklist was evaluated by local experts (two gastroenterologists, one gastroenterology nurse practitioner, and five hospitalists) using a modified Delphi method $^{15}$ and further edited to reflect best practices at our institution. The PCAT also includes two global measures: a global skill assessment scale and an entrustment scale. We included these subjective but 
important measures because global ratings and checklists do not always correlate and each type of measure has its own strengths and weaknesses. ${ }^{8,9,13}$ Thus, we adopted the hybrid approach ${ }^{13}$ and combined the checklist with two global measures. We modified the five-item global skills assessment scale of Sawyer and colleagues ${ }^{13}$ to a four-item scale that better captured different levels of performance a resident might realistically achieve. Namely, with limited procedure experience, it is unlikely that residents would perform at the expert level. The modified global skills assessment scale ranges from novice to proficient. We included an entrustment scale as its own separate tool component as this is a common assessment construct in competency-based medical education and is similar to the way we assess residents in our own assessment system. ${ }^{16}$ The PCAT was used for formative feedback. It was also used as a summative measure in that if a learner missed any critical steps in the checklist, it was considered an automatic failure, with the missed step(s) requiring completion prior to passing the simulation. After the final simulation assessment, learners were required to achieve the Minimum Passing Standard, which was a checklist score of 19 out of 26 , a global score of 2 out of 4 , and an entrustment score of 2 out of 5 . The Minimum Passing Standard represented the minimum level of competence needed before a learner received permission to perform the procedure on actual patients under direct supervision. The aggregate score of a checklist score of 23 out of 26 , a global score of $\geq 3$ out of 4 , and an entrustment score of $\geq 4$ out of 5 signified the ability of a learner to perform the procedure without supervision. Establishment of these scores was accomplished through standard-setting procedures with local and national experts.

Debriefing

Debriefing was completed after each evaluation. The instructor reviewed the assessment with the learner to discuss any deficiencies that existed and identified areas of needed improvement. The learner then had the opportunity to practice deficiencies as described above.

\section{Results}

The comprehensive educational curriculum including the initial paracentesis simulation experience was completed by a total of 123 internal medicine and medicine-pediatric residents. Initial simulation training for all residents took approximately 4 months to complete. Of these residents, over $80 \%$ (99 out of 123) accessed the educational material including the presentation, videos, and assessments. The materials were accessed on a total of 1,196 occasions. The average scores on the ultrasound and paracentesis quizzes were $76.1 \%$ and $75.3 \%$, respectively. All residents achieved above $70 \%$; however, the assessments were set up such that an individual learner could repeat the quizzes multiple times in order to achieve a passing score.

A total of 18 hospitalist faculty members expressed interest in helping with training after the curriculum was presented at a department meeting. Once simulation dates were set, the free online service SignUpGenius was used for faculty to self-select sessions that would fit best with their schedules. Each faculty member participated in two to three half-day sessions as well as one training session.

All residents achieved the Minimum Passing Standard after their initial training session. Learners varied in the amount of times they needed to repeat an individual checklist step, either because they wanted more practice or because the instructor felt they should repeat that step. However, all residents completed all steps to their and the instructor's satisfaction within the allotted 1-hour session. In general, aggregate scores on the PCAT increased with increasing PGY level, although there was not a large difference between PGY 2s and PGY 3s. The number of checklist items performed correctly increased with each year in PGY level, with PGY 3s completing over $88 \%$ of the checklist. Approximately $10 \%$ of PGY 1s, $50 \%$ of PGY 2s, and $50 \%$ of PGY 3s achieved the aggregate PCAT score signifying competence for unsupervised practice.

Postcurricular feedback was overwhelmingly positive. Faculty indicated their involvement to be a positive experience and felt that teaching the procedure served as a good refresher for their own paracentesis performance. Residents anecdotally reported an increase in self-confidence and competence in obtaining 
informed consent, using ultrasound to identify ascites, and performing a paracentesis. On the annual anonymous program-evaluative survey, the paracentesis training curriculum was rated as the fourth highest learning experience out of our 14 core curricular activities. On average, when asked to rate the quality of the learning experience, residents rated the paracentesis program as good (average: 3.88 on a scale from $1=$ worst possible to $5=$ great; $n=86$ ). Select narrative comments included the following:

- "Great paracentesis program ... duplicate with other procedures."

- "I would love to have more procedure training, perhaps [similar sessions] for each major type of procedure we do including central line, lumbar puncture and thoracentesis."

- "Make more procedure practice sessions!"

- "This is great! We need more of it."

"While certainly simulation cannot replace real practice, I found these sessions to be very useful in gaining familiarity and comfort with the tools and ultrasound, and a good way to remind me of the critical pieces of the procedure (especially when it has been a while). Overall, very useful, would very much like to extend it to central line."

\section{Discussion}

We view our curriculum as a new strategy in the education of bedside procedures, offering a comprehensive approach to address some of the current inadequacies in procedural education. This multifaceted method offers both cognitive and psychomotor education using techniques designed to provide the long-term knowledge retention needed in clinical practice. We believe the strengths of this curriculum are related to its comprehensive approach to procedure education with the goal to measure and attain competence. In general, we followed the "learn, see, practice, prove, do, maintain" model ${ }^{13}$ in creating this curriculum. We believe that this model creates a more systematic and reliable way to teach procedural skills in order to ensure competency. Learners begin to learn via the preprocedural education and designed cases. The incorporation of multiple teaching techniques (i.e., preprocedural self-learning and small-group problem-based learning with the cases) offers the benefit of education through a variety of media. We prefer to spread these teaching modules with simulation across a prolonged time period, offering the benefit of the spacing effect, where information is presented and repeated during spaced intervals of time. This strategy of learning has been shown to create more long-term retention of knowledge. ${ }^{17}$ The seeing component is completed with the paracentesis video. Learners are able to practice by simulation. We create an environment for deliberate practice ${ }^{18}$ by offering learners a goaloriented time to practice with repetition as well as direct and immediate feedback on performance by the instructors. Learners can then incorporate feedback in real time to improve their performance and make continued adjustments as needed. We find practice to be extremely valuable as learners are able to take their time with each component of the procedure and ask questions they have not had time or have been unable to ask during their experience on the wards. Furthermore, learners are able to try several techniques and decide which feels most comfortable, something that cannot be done without simulation. Learners then prove a minimum level of competence via the evaluated simulation component, after which point they can safely do paracentesis procedures with supervision during their hospital rotations. Finally, learners return to the simulation lab and review clinical cases to maintain their knowledge and skills, again offering the spacing technique for long-term retention of procedural skills. We view this opportunity for maintenance of skill to be crucial since learners often go prolonged periods of time without performing many procedures, which results in deterioration of procedural skill and significant opportunity for error.

It is imperative to note that while the assessment tool has a standard score for the ability to competently perform the procedure independently, it would not be recommended that a learner who achieves this score in the simulation setting be permitted to perform a paracentesis on a live patient without supervision. 
Although the optimal number of observations is not clear, it is likely that multiple observations showing competence on simulated and live procedures would need to be obtained prior to making summative decisions about independent practice. Scores on the PCAT are meant to be tracked over time to monitor learner progress. These data may help with milestone reporting to the Accreditation Council for Graduate Medical Education.

All data and feedback from residents and faculty alike indicated a well-received curriculum. Preprocedural material was completed appropriately and was in fact accessed multiple times by many of the learners for review. As expected, learners' skills and competence improved as their level of training progressed. However, many who by old definitions of competence would have been deemed able to perform the procedure unsupervised (i.e., they had completed three paracentesis procedures) were not deemed competent to perform the procedure unsupervised via this new assessment construct. Anecdotally, this was perceived as a strong moment of learning as it created cognitive dissonance within the learner and encouraged him or her to take repeated simulations sessions and future case discussions seriously. While we do not yet have full, analyzed data for performance during repeat simulation sessions, the sentiment from learners was clear: Repeated opportunities to practice the procedure with feedback over time helped improve self-confidence and self-competence. Since it is likely that skill declines over time, we suggest this approach to others as a template for how one might approach procedure education, but the optimal time for repeated simulation sessions is unclear. We hope to replicate this multifaceted approach, as learner feedback consistently mentioned the desire to expand the curriculum to include other invasive bedside procedures.

A major challenge initially was scheduling learners and faculty. By placing one chief resident in charge of scheduling, we were able to better keep track of each learner's participation so we knew when to schedule him or her in the simulation lab for his or her next session. Using an online sign-up link helped ensure faculty were present for each session. Based on the limited availability of resources, moving forward we are going to try to better integrate the repeated simulation sessions when residents complete their gastroenterology rotations. We also will be using the PCAT in the live patient environment when faculty and fellows supervise paracentesis procedures. We will need to elicit feedback from learners and faculty to see what their experience using the PCAT is in the live environment.

This comprehensive curriculum and associated data have several limitations. First, the PCAT assessments were completed on simulated paracenteses and not those done on live patients. Next steps include using the PCAT on live patient procedures to help track progress over time and structure formative feedback. Second, we could not that ensure learners completed the didactic materials prior to the simulation sessions. These materials were meant to be mandatory, but due to faculty and simulation lab time constraints, we could not reschedule the $20 \%$ of learners who did not complete the online modules. It is possible that this affected performance during the actual simulation sessions. Third, we did not measure baseline, first run-through scores because it had already been shown that simulation training increased learner skill in performing procedures and we did not want to spend our limited simulation time having learners struggle through portions of the procedure incorrectly. In addition, all learners watched a goldstandard demonstration prior to performing the procedure, so we thought this would alter the integrity of baseline assessments. Fourth, we do not have return scores to share at this time and thus do not know the optimal retraining interval. While it would be important to know that training is durable, skill degradation is a complex topic. Our purpose for retraining was not to prove that training was durable but rather to ensure repeated deliberate practice over time in anticipation of skill decay. Further research might be aimed at determining the optimal time of such retraining. Although we chose 3- to 6-month intervals, another approach might be to train right before a procedural rotation. Our main goal for the medical community is 
to share a potential framework for repeated training, but we do not mean to require that others must follow this sequencing.

This curriculum is a valuable resource that could be utilized by other programs that require paracentesis training (e.g., emergency medicine residency and gastroenterology fellowship programs). Future opportunities exist to use this curricular approach for other invasive bedside procedures. Specifically, for internal medicine, our goals include a future curriculum for central venous catheter and arterial line insertion.

Dana Sall, MD, MEd: Assistant Professor, Department of Medicine, University of Cincinnati Medical Center; Associate Program Director, Internal Medicine Residency Program, University of Cincinnati Medical Center

Gregory W. Wigger, MD: Resident Physician, Department of Medicine, University of Cincinnati Medical Center

Benjamin Kinnear, MD: Associate Program Director, Internal Medicine-Pediatrics Residency Program, University of Cincinnati College of Medicine; Assistant Professor, Department of Medicine, University of Cincinnati College of Medicine; Assistant Professor, Department of Pediatrics, Cincinnati Children's Hospital Medical Center

Matthew Kelleher, MD, MEd: Associate Program Director, Internal Medicine-Pediatrics Residency Program, University of Cincinnati College of Medicine; Assistant Professor, Department of Medicine, University of Cincinnati College of Medicine; Assistant Professor, Department of Pediatrics, Cincinnati Children's Hospital Medical Center

Eric Warm, MD: Professor, Department of Medicine, University of Cincinnati Medical Center; Program Director, Internal Medicine Residency Program, University of Cincinnati Medical Center

Jennifer K. O'Toole, MD, MEd: Program Director, Internal Medicine-Pediatrics Residency Program, University of Cincinnati College of Medicine; Associate Professor, Department of Pediatrics, Cincinnati Children's Hospital Medical Center; Associate Professor, Department of Internal Medicine, University of Cincinnati College of Medicine

\section{Disclosures:}

None to report.

\section{Funding/Support:}

Dr. O'Toole reports personal fees from the I-PASS Patient Safety Institute during the conduct of the study.

Informed Consent:

All identifiable persons in this resource have granted their permission.

Ethical Approval:

Reported as not applicable.

\section{References}

1. Duffy FD, Holmboe ES. What procedures should internists do? Ann Intern Med. 2007;146(5):392-393. https://doi.org/10.7326/0003-4819-146-5-200703060-00012

2. Rodriguez-Paz JM, Kennedy M, Salas E, et al. Beyond "see one, do one, teach one": toward a different training paradigm. BMJ Qual Saf. 2009;18(1):63-68. https://doi.org/10.1136/qshc.2007.023903

3. American Board of Internal Medicine. Policies and Procedures for Certification. Philadelphia, PA; American Board of Internal Medicine: 2017

4. Augustine EM, Kahana M. Effect of procedure simulation workshops on resident procedural confidence and competence. J Grad Med Educ. 2012;4(4):479-485. https://doi.org/10.4300/JGME-D-12-00019.1

5. Barsuk JH, Cohen ER, Vozenilek JA, O’Connor LM, McGaghie WC, Wayne DB. Simulation-based education with mastery learning improves paracentesis skills. J Grad Med Educ. 2012;4(1):23-27. https://doi.org/10.4300/JGME-D-11-00161.1

6. Fincher R-ME. Procedural competence of internal medicine residents: time to address the gap. J Gen Intern Med. 2000;15(6):432-433. https://doi.org/10.1046/j.1525-1497.2000.0004010.x

7. Lenchus JD, Carvalho CM, Ferreri K, et al. Filling the void: defining invasive bedside procedural competency for internal medicine residents. J Grad Med Educ. 2013;5(4):605-612. https://doi.org/10.4300/JGME-D-13-00030.1

8. Huang GC, Newman LR, Schwartzstein RM, et al. Procedural competence in internal medicine residents: validity of a central venous catheter insertion assessment instrument. Acad Med. 2009;84(8):1127-1134

https://doi.org/10.1097/ACM.0b013e3181acf491 
9. Walzak A, Bacchus M, Schaefer JP, et al. Diagnosing technical competence in six bedside procedures: comparing checklists and a global rating scale in the assessment of resident performance. Acad Med. 2015;90(8):1100-1108. https://doi.org/10.1097/ACM.0000000000000704

10. Smith CC, Gordon CE, Feller-Kopman D, et al. Creation of an innovative medical procedure service and a method to evaluate house staff competency. J Gen Intern Med. 2004;19(5):510-513. https://doi.org/10.1111/j.1525-1497.2004.30161.x

11. Thomsen TW, Shaffer RW, White B, Setnik GS. Paracentesis. N Engl J Med. 2006;355(19):e21. https://doi.org/10.1056/NEJMvcm062234

12. Ma I, Sharma N, Nagassar S, Wishart I, Holroyd-Leduc J, Novak K. Ultrasound-guided paracentesis. MedEdPORTAL. 2014;10:9774. https://doi.org/10.15766/mep_2374-8265.9774

13. Sawyer T, White M, Zaveri P, et al. Learn, see, practice, prove, do, maintain: an evidence-based pedagogical framework for procedural skill training in internal medicine. Acad Med. 2015;90(8):1025-1033. https://doi.org/10.1097/ACM.0000000000000734

14. Riesenberg LA, Berg K, Berg D, et al. The development of a validated checklist for paracentesis: preliminary results. Am J Med Qual. 2013;28(3):227-231. https://doi.org/10.1177/1062860612460399

15. de Villiers MR, de Villiers PJT, Kent AP. The Delphi technique in health sciences education research. Med Teach. 2005;27(7):639-643. https://doi.org/10.1080/13611260500069947

16. Warm EJ, Mathis BR, Held JD, et al. Entrustment and mapping of observable practice activities for resident assessment. J Gen Intern Med. 2014;29(8):1177-1182. https://doi.org/10.1007/s11606-014-2801-5

17. Kerfoot BP, Fu Y, Baker H, Connelly D, Ritchey ML, Genega EM. Online spaced education generates transfer and improves longterm retention of diagnostic skills: a randomized controlled trial. J Am Coll Surg. 2010;211(3):331-337. https://doi.org/10.1016/j.jamcollsurg.2010.04.023

18. Ericsson KA. Deliberate practice and acquisition of expert performance: a general overview. Acad Emerg Med. 2008;15(11):988994. https://doi.org/10.1111/j.1553-2712.2008.00227.x

Received: April 29, 2018 | Accepted: July 31, 2018 | Published: August 30, 2018 